\title{
Interactive comment on "Statistical analyzing the effect of ionospheric irregularity on GNSS radio occultation atmospheric measurement" by Mingzhe Li and Xinan Yue
}

\section{Mingzhe Li and Xinan Yue}

limingzhe92@hotmail.com

Received and published: 20 February 2021

Response to Reviewer 2 Thanks for the invaluable comments and suggestions that helped to improve the quality of this manuscript. Considering all the comments, pointto-point responses are addressed in this rebuttal as follows.

General comment: This manuscript investigated the effect of ionospheric irregularity on atmospheric RO data. Specifically, the authors took the S4 index as a proxy of the occurrence of ionospheric irregularity, and examined two atmospheric metrics - failure rate of atmospheric $\mathrm{RO}$ retrievals, and bending angle oscillation. Through comparing the morphology and seasonal dependency of two atmospheric metrics with the ones 
from irregularity occurrence, the authors demonstrated the pattern similarity between them, which indicated the impact of irregularity on the atmospheric retrievals. It is overall an interesting study and well written. The topic fits well to the scope of the AMT. Therefore, I would recommend this manuscript for publication after a few minor revisions.

Reply: We have checked and revised the manuscript. Thank you very much for the helpful comments.

Specific Comments: L144: LC $\rightarrow$ ionospheric-corrected LC

Reply: Thanks for the comment. It has been revised as "ionospheric-corrected LC".

L217-218: The occurrence rates of Es and FI are mixed together in this study, which makes the interpretation of results a bit complicated, though the authors already tried their best to explain the impact of Es and FI separately in the text. What if you do the occurrence rate analysis separately for Es and FI based on the altitude of the S4 maximum?

Reply: Thanks for the comment. On condition that the morphologies of the failed inverted $\mathrm{RO}$ events and the bending angle oscillation are related to both Es and $\mathrm{FI}$, we plot the occurrence rate of Es and FI together for discussion. For convenient comparison, a figure has been added as the new Figure 2 to display the occurrence rates of Es and FI separately. Please note that the serial numbers of figures after Figure 1 have been changed in orders.

L288-292: Is there any proof to support the statement that the FI occurrence plays the main role in the failed inverted $\mathrm{RO}$ occurrence?

Reply: Thanks for the comment. It is a guess made by us. We are sorry for our neglect here. The related description has been removed. We hope to study the causing of failed inverted $\mathrm{RO}$ events and bending angle oscillation in high latitudes more specifically in our further study. 
Beside the large ionospheric residual caused by ionospheric irregularity, atmospheric RO data can be identified as "Bad" for many different reasons. For example, over the AMTD time period selected in this manuscript, 2011-2013, there are couple of sudden warming events happened, which makes the atmospheric structure changes significantly and far from the climatology, especially over the polar winter. The RO retrieval could be "Bad" because of the large deviation from the climatology under such condition.

Interactive comment

Reply: Thank you very much for the comment. In this paper we mainly concentrate on studying the correlation between the failed inverted $\mathrm{RO}$ events and the ionospheric small-scale irregularity. As your suggestion, the sudden warming events could be an important reason for the failed inverted $\mathrm{RO}$ events. It is essential for us to investigate its effect on the RO bending angle.

Fig. 2: What if the figure just focuses on the low and mid-latitude herein since you already presented the polar results in Fig. 3? Also, you might consider to use the discrete color bar instead of the gradient one. Fig. 3. This is a polar coordinate, not a Cartesian one. It's better to delete "MLAT" and "MLT" herein.

Reply: Thanks for the comment. As depicted in the new Figure 5, the correlations between the failed inverted $\mathrm{RO}$ events and the ionospheric irregularity, are not as good as those in low and middle latitudes. That's the reason why we mainly concentrate on the study in low and middle latitudes in the following part in this manuscript. We hope to study the causing of failed inverted $\mathrm{RO}$ events and bending angle oscillation in high latitudes more specifically in our further work. Both the ionospheric irregularity effects and the sudden warming event effects should be taken into consideration. The colorbars of all figures have been revised and the "MLAT" and "MLT" in the new Figure 4 has been deleted.

Interactive comment on Atmos. Meas. Tech. Discuss., doi:10.5194/amt-2020-440, 2020.

Printer-friendly version

Discussion paper 
AMTD
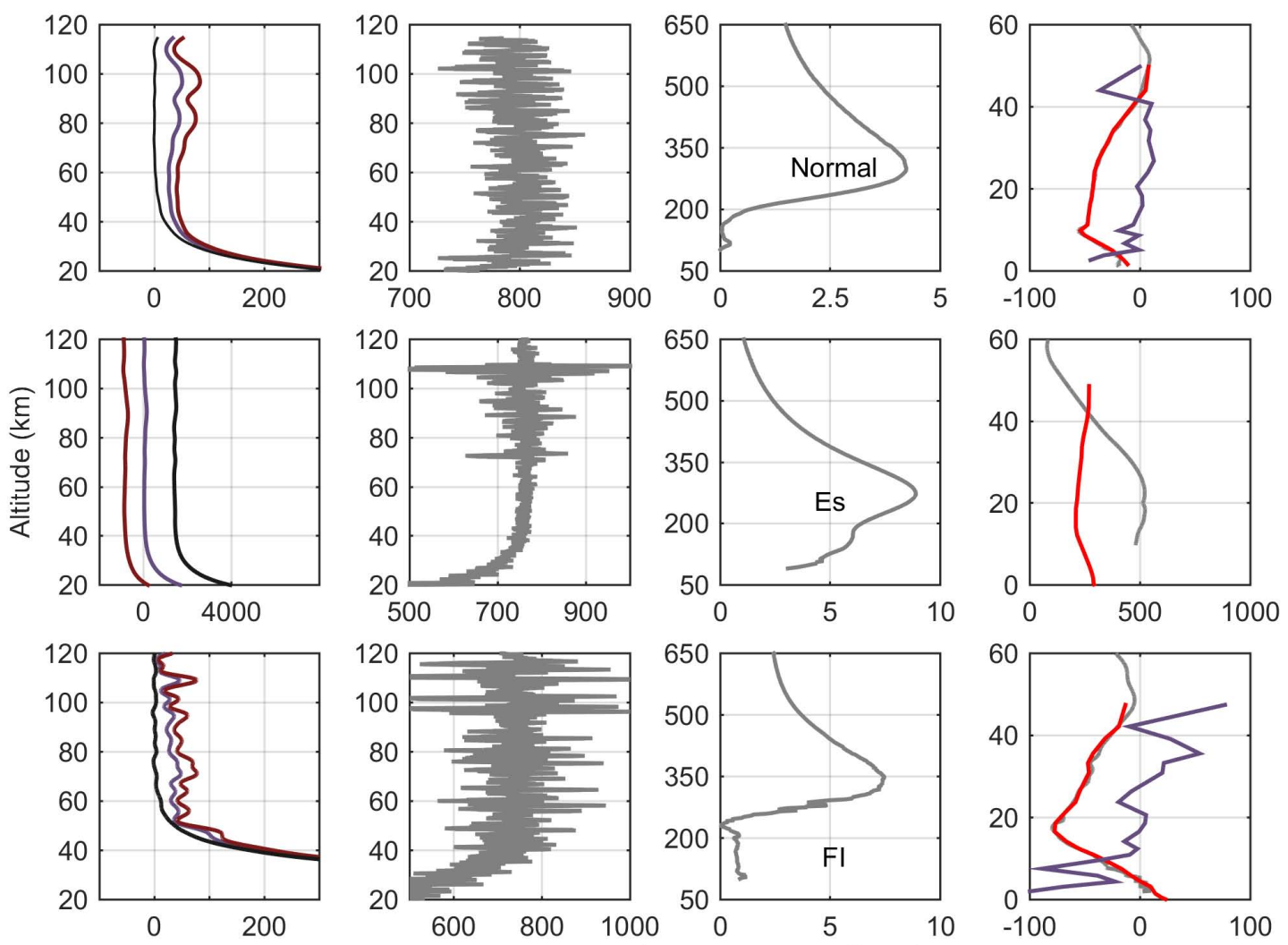

$\begin{array}{lll}\text { (a) Bending angle }[\mu \mathrm{rad}] & \text { (b) L1 C/A SNR }\left[V V^{-1}\right] & \text { (c) } \mathrm{Ne}\left[10^{5} \mathrm{~cm}^{-3}\right]\end{array}$

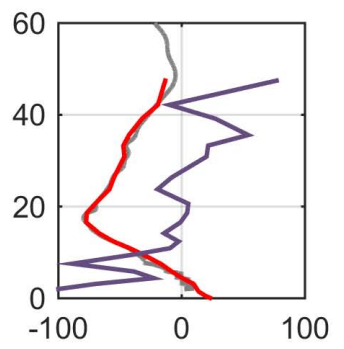

(d) Temperature $\left[{ }^{\circ} \mathrm{C}\right]$

Printer-friendly version

Interactive comment

Fig. 1.

Discussion paper 
AMTD
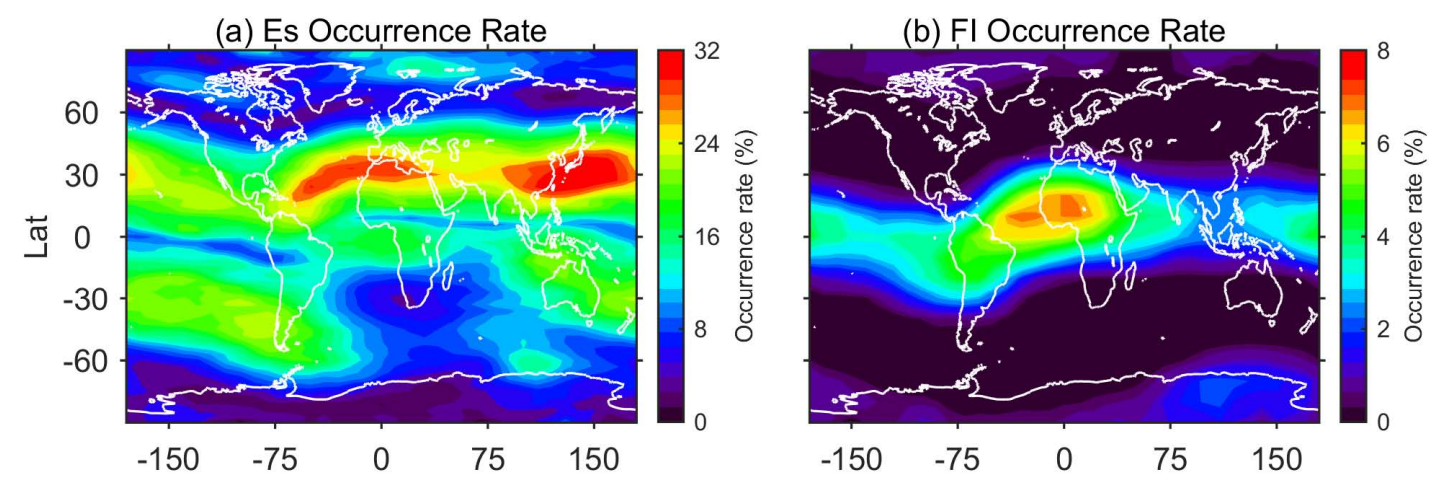

\section{Interactive comment}
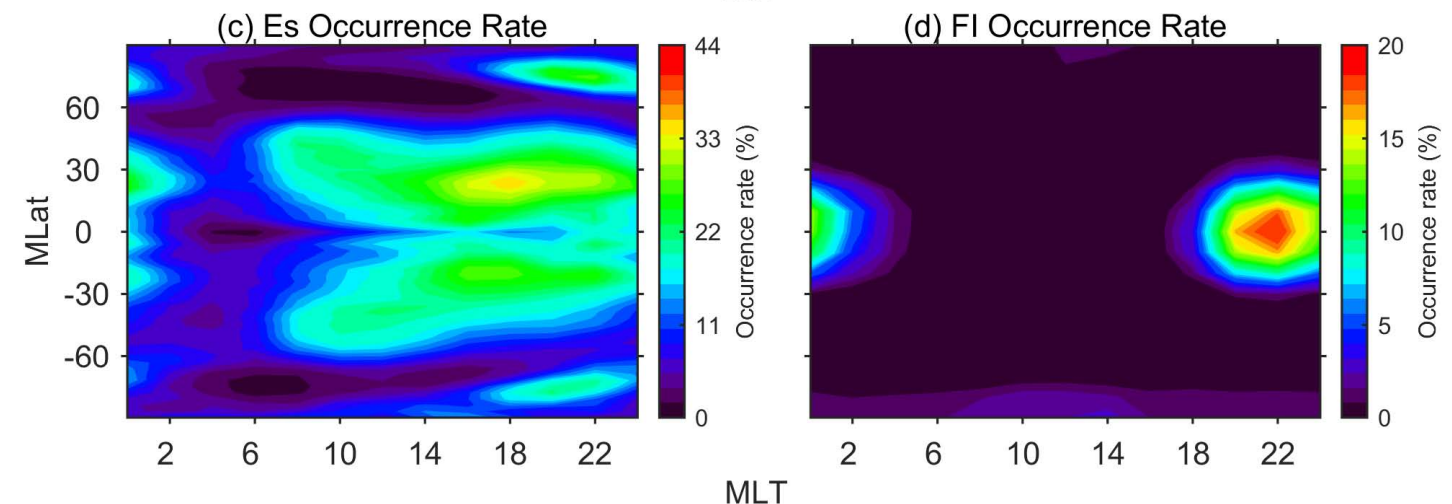

Fig. 2. 
AMTD

Interactive comment
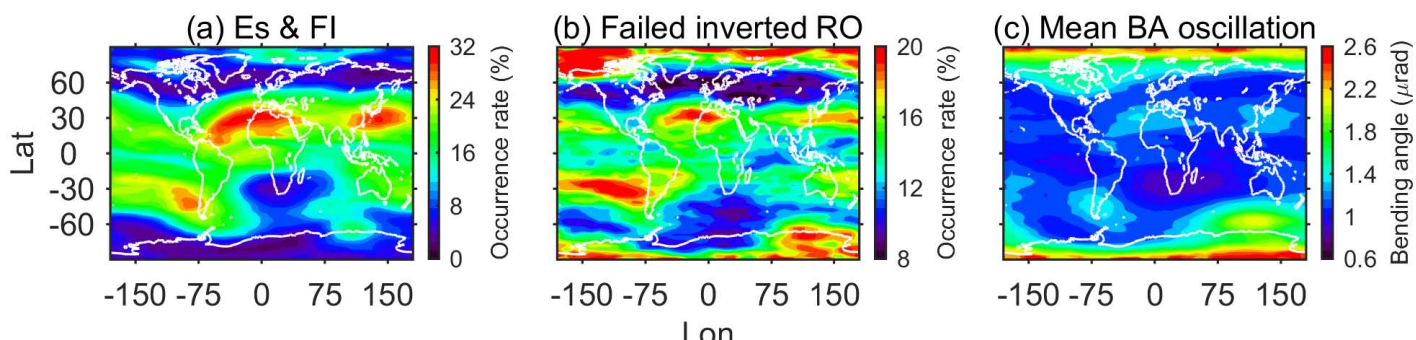

(d) Es \& FI
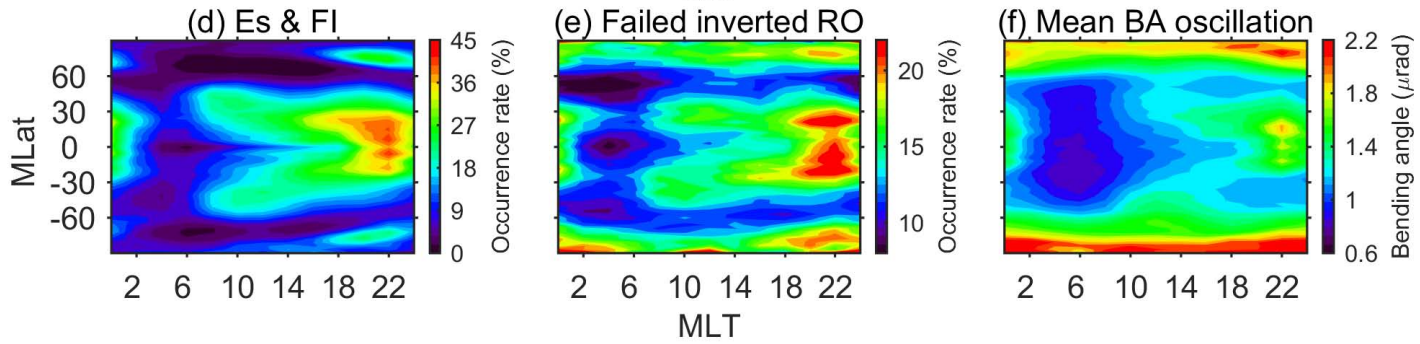

$\begin{array}{llllll}2 & 6 & 10 & 14 & 18 & 22\end{array}$

Fig. 3. 
(a) Es \& FI

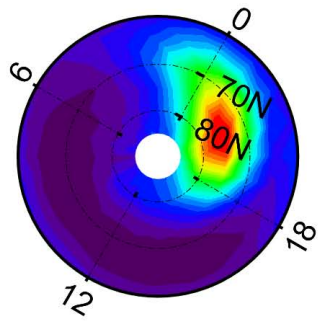

(d) Es \& FI

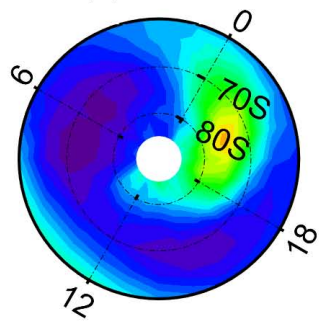

(b) Failed inverted RO
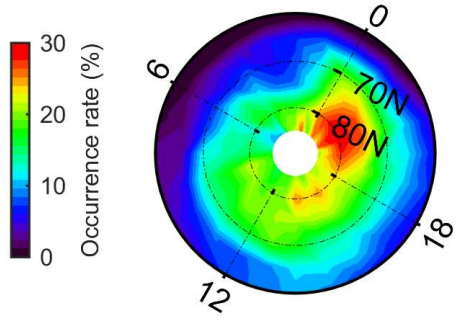

(e) Failed inverted RO
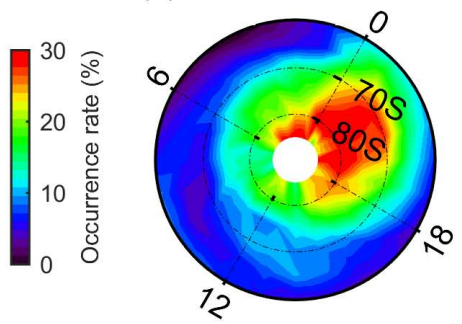

(c) Mean BA oscillation

Interactive comment
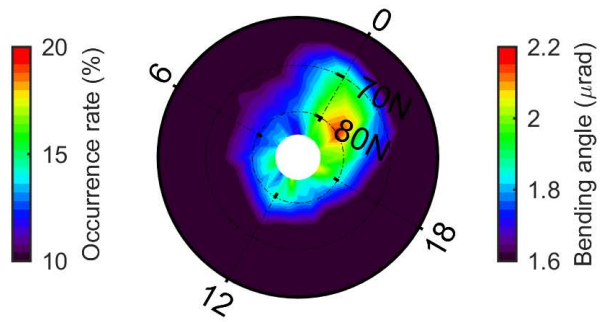

Fig. 4.

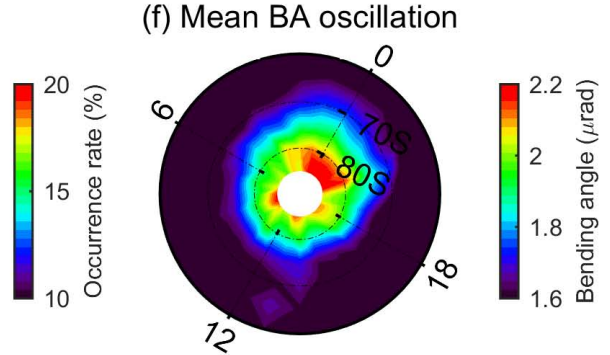


AMTD
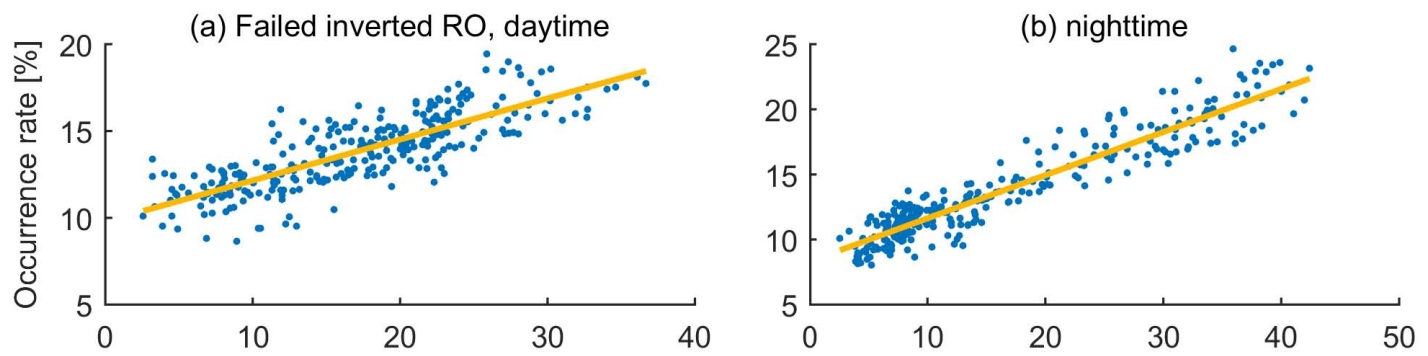

Interactive comment
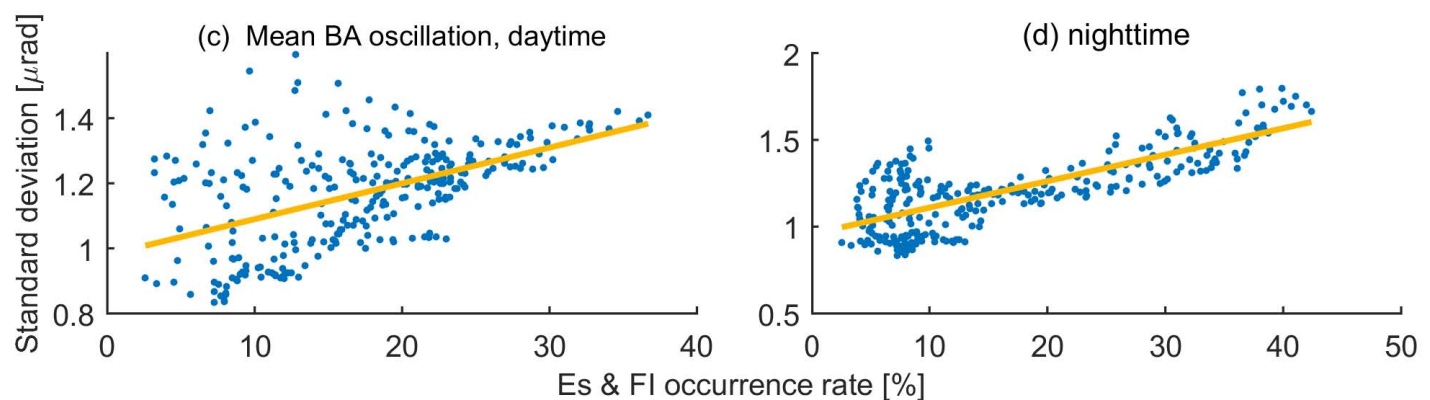

Fig. 5. 


\section{AMTD}

(a) Es \& FI, Equinox
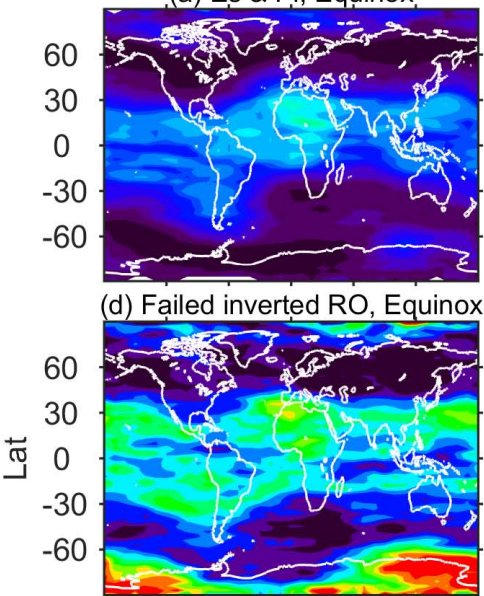

(g) Mean BA oscillation, Equinox

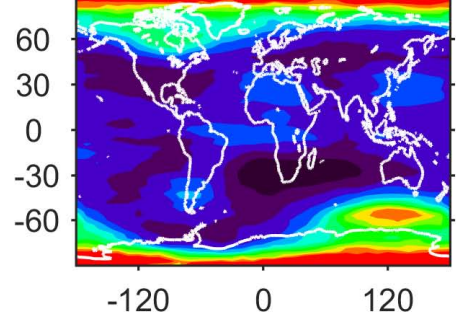

(b) Northern Summer

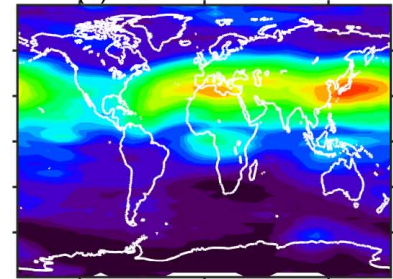

(e) Northern Summer

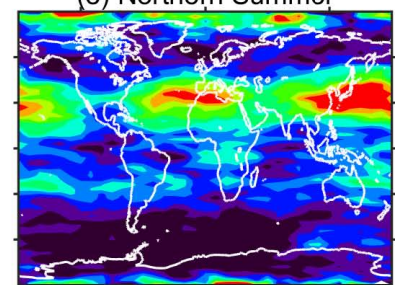

(h) Northern Summer

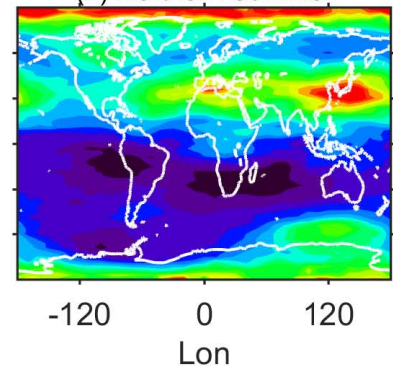

(c) Northern Winter

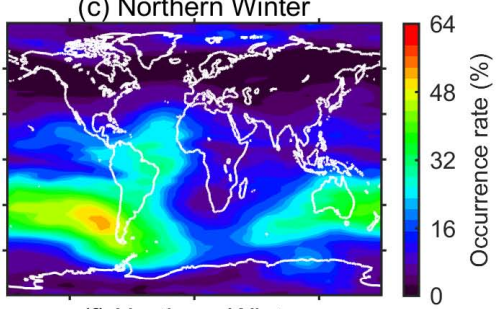

(f) Northern Winter

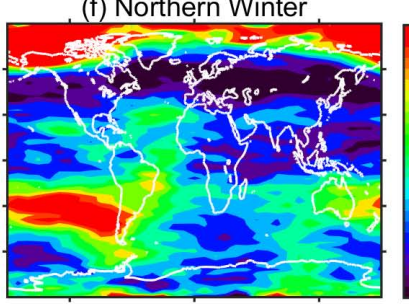

(i) Northern Winter

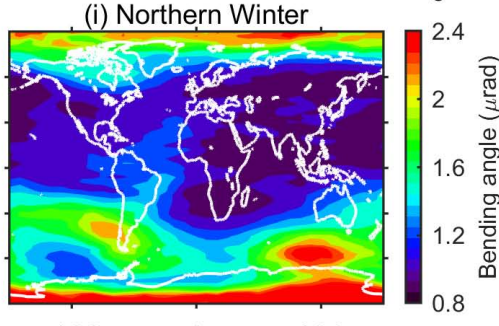

$\begin{array}{lll}-120 & 0 & 120\end{array}$
Interactive

comment

Fig. 6.

Printer-friendly version

Discussion paper 
AMTD
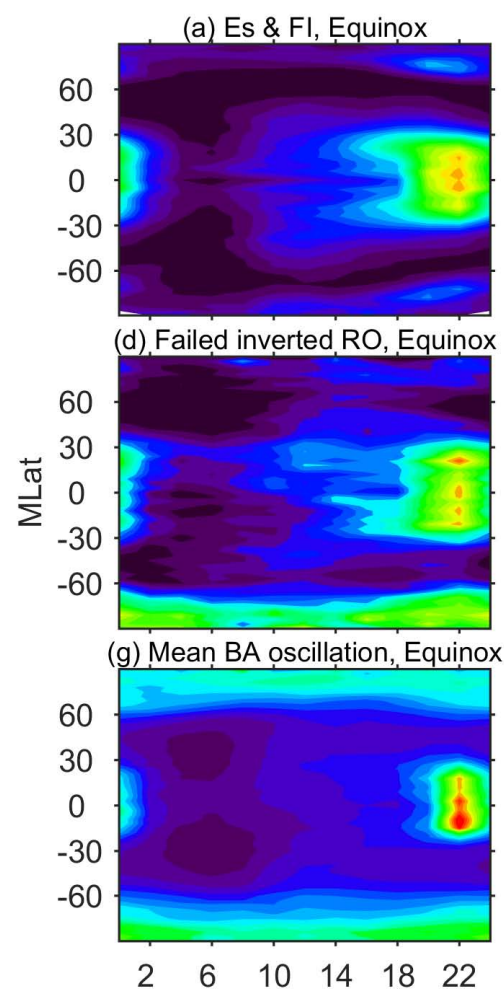

(b) Northern Summer

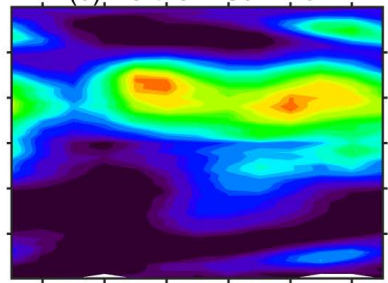

(e) Northern Summer

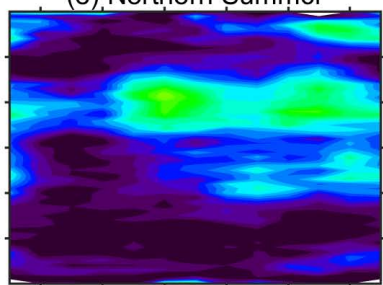

(h) Northern Summer

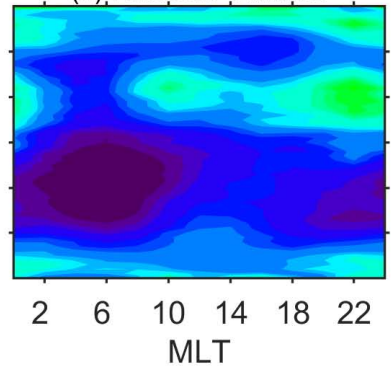

(c) Northern Winter

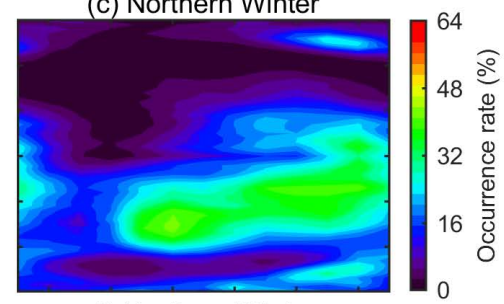

(f) Northern Winter

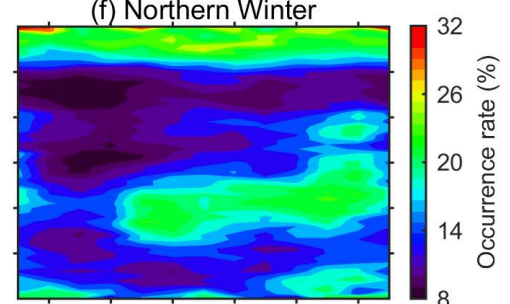

(i) Northern Winter

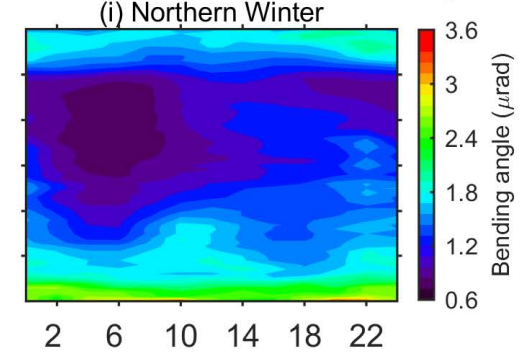

$\begin{array}{llllll}2 & 6 & 10 & 14 & 18 & 22\end{array}$

Interactive comment

Fig. 7. 